\title{
A case of emergency pancreatoduodenectomy for bleeding from the duodenal mucosa due to arteriovenous malformation of the pancreatic head
}

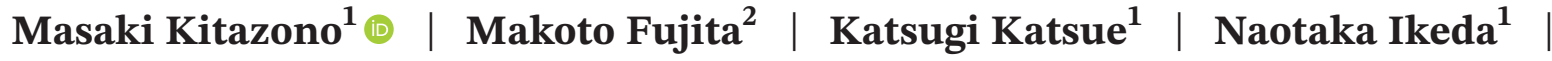 \\ Tomohiro Oyama $^{1}$ | Mayumi Eguchi ${ }^{1}$ | Go Kamimura ${ }^{1}$ | Rikiya Sato ${ }^{1}$ | \\ Shuichiro Uchiyama $^{1}$ | Ryoichi Toyosaki ${ }^{1}$ | Toyokuni Suenaga ${ }^{1}$
}

${ }^{1}$ Department of Surgery, Public Interest Incorporated Association, Kagoshima Kyosaikai, Nanpuh Hospital,

Kagoshima City, Japan

${ }^{2}$ Division of Medical Support, Public Interest Incorporated Association, Kagoshima Kyosaikai, Nanpuh Hospital, Kagoshima City, Japan

\section{Correspondence}

Masaki Kitazono, Department of Surgery, Public Interest Incorporated Association, Kagoshima Kyosaikai, Nanpuh Hospital, 14-3, Nagata Cho, Kagoshima City, Kagoshima Pref. 8928512, Japan.

Email: opera_2@hotmail.com

\begin{abstract}
A rare case of bleeding from the duodenal mucosa due to arteriovenous malformation of the pancreatic head is reported. Caution needs to be observed, then excision may be necessary since noninvasive treatment such as coil embolization is not enough for complete hemostasis when patient suddenly decompensates.

\section{K E Y W O R D S}

duodenal bleeding, pancreatic arteriovenous malformation
\end{abstract}

\section{1 | INTRODUCTION}

Arteriovenous malformation of the pancreatic head is a relatively rare disease that can cause gastrointestinal bleeding and abdominal pain and requires appropriate and prompt treatment. Herein, we discuss the clinical presentation and management of a patient with arteriovenous malformation of the pancreatic head.

Arteriovenous malformation of the pancreatic head (Ph-AVM) is relatively rare, causing gastrointestinal and intra-abdominal hemorrhage and severe abdominal pain. We herein report a case of Ph-AVM with hemorrhagic shock due to massive duodenal bleeding, successfully treated by emergency pancreatoduodenectomy.

\section{2 | CASE PRESENTATION}

A 45-year-old man with a 20-year-old history of duodenal ulcer presented to our hospital with vomiting, lightheadedness, and melena. There was no history of other gastrointestinal conditions, cardiovascular conditions, pancreatitis, infection, trauma, or family history. Two days before the admission, he first experienced melena following a heavy meal. At the time of admission, there was no abnormality with the physical examination; however, laboratory data showed anemia (hemoglobin of $8.7 \mathrm{~g} / \mathrm{dl}$ and red blood cell count of 2.89 million/ $\mu \mathrm{l}$ ), high white

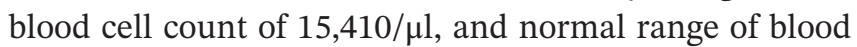
pressure on both systolic $\mathrm{BP}(\mathrm{SBP})$ and diastolic $\mathrm{BP}(\mathrm{DBP})$. 
The next day, he presented with hemorrhagic shock, as his SBP decreased down to $68 \mathrm{mmHg}$, due to vasovagal reflex, right after he had melena. The patient was managed with urgent transfusion immediately, and the emergency endoscopy performed did not identify the source of upper gastrointestinal bleeding. Computed tomography $(\mathrm{CT})$ revealed dilated blood vessels in the lower part of the descending duodenum and spotty staining of the pancreatic uncinate process at the early phase (Figure 1), and the next day multifocal bleeding from the edematous duodenal mucosa near the papilla of Vater (Figure 2) were observed with second-time endoscopy. Considering the possibility of bleeding due to vascular lesions such as $\mathrm{Ph}-\mathrm{AVM}$ based on CT findings and endoscopic findings, interventional radiology was performed for further diagnosis and treatment. Angiography revealed the definitive diagnosis of Ph-AVM with abnormal reticular blood vessels in the descending limb of the duodenum (Figure 3). Coil embolization was performed, but unfortunately did not achieve complete hemostasis, thus we decided to perform emergency surgery. A typical pancreatoduodenectomy was performed 3 days after the definitive diagnosis. Surgical findings showed the proliferation of abnormally dilated blood vessels at the pancreatic head. The surgery time was $5 \mathrm{~h}$, which is the average time for this type of surgery with experienced surgeons and the amount of bleeding was $500 \mathrm{ml}$.

Histopathological findings showed collections of irregularly tortuous blood vessels within the thick wall of the duodenal proper muscle layer adjacent to the pancreatic parenchyma near the major duodenal papilla. Fibrin thrombi were occasionally seen in the pancreatic parenchyma and submucosa of the duodenum (Figure 4).

The postoperative course was uneventful. However, preoperative, it was noted that patient's SBP always decreased down remarkably, every time after he had melena or vomiting. Nevertheless, it is clear that patient's SBP no longer went under $110 \mathrm{mmHg}$ as there was no bleeding after the surgery. The total hospital length of stay (LOS) was 32 days, and after this lifesaving surgery, and after postoperative care and follow-up care, the patient was discharged home and he has remained in excellent health, so far, for 4 years and 9 months.

\section{DISCUSSION}

Pancreatic AVM, first reported by Halpern et al. in $1968,{ }^{1}$ is one of the abnormal blood flow diseases in which the arteriovenous system is short-circuited and anastomosed in the pancreas. The most common sites for gastrointestinal arteriovenous malformations are the cecum, ascending colon, and jejunum, and pancreatic AVM accounts for
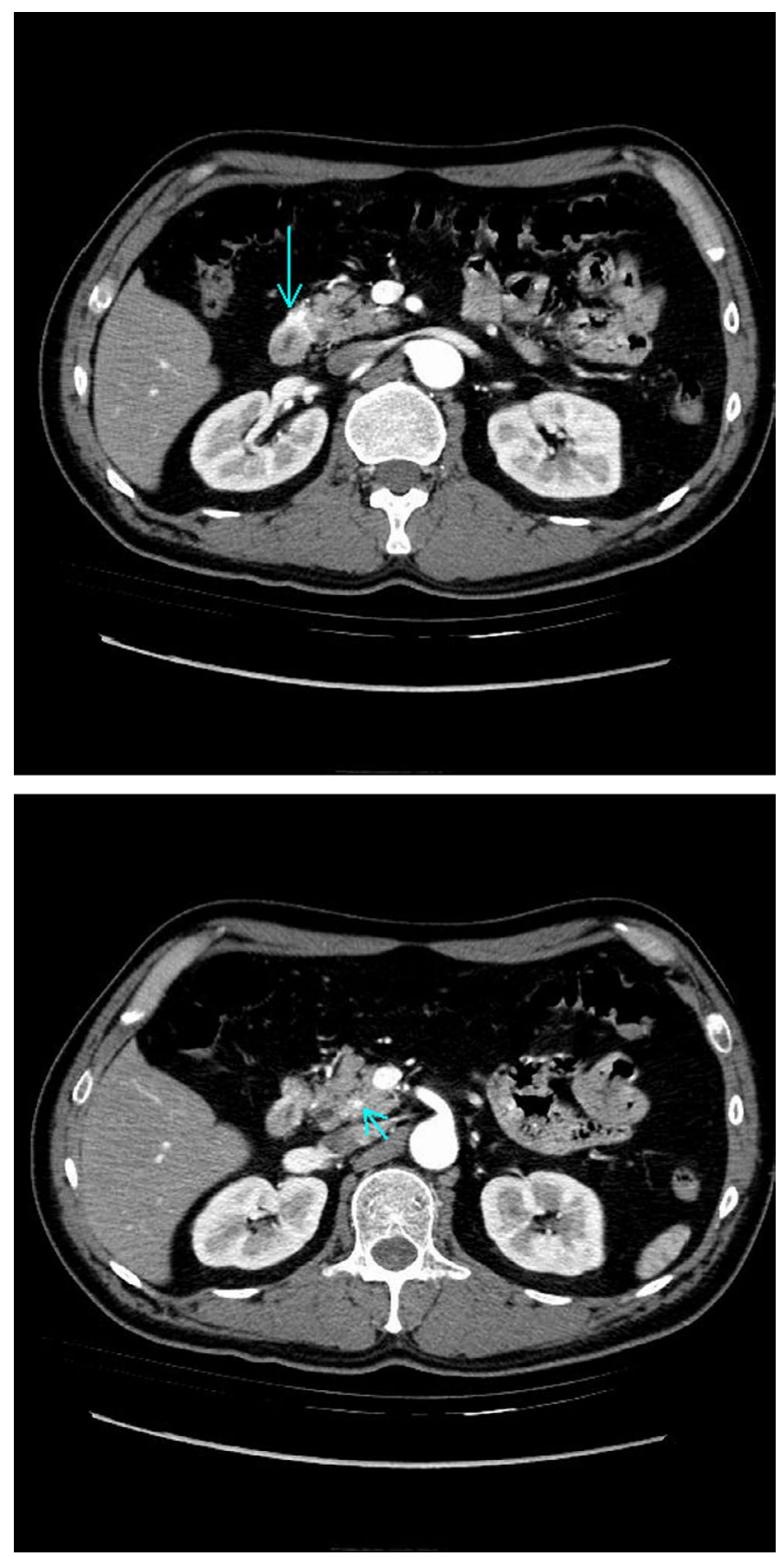

F I G U R E 1 Abdominal CT examination: Dilated blood vessels in the lower part of the descending duodenum, and spotty staining of the pancreatic uncinate process at the early phase were observed

only $0.9 \% .^{2}$ The causes of this disease are classified into congenital due to the remnants of the primitive vascular network, ${ }^{3}$ and acquired due to excessive angiogenesis caused by inflammation such as pancreatitis and trauma. ${ }^{4}$ In this case, it was considered to be congenital because there was no particular history in the past.

According to what was reported by Song KB et al. in $2012,{ }^{5}$ pancreatic AVM is an overwhelmingly male disease, since there were males (88.4\%) predominantly opposed to females, as shown in this case. The median age at diagnosis was 50 years of age, which is relatively young. 
FIG URE 2 Upper gastrointestinal endoscopy: Multifocal bleeding from the edematous duodenal mucosa near the papilla of Vater was observed

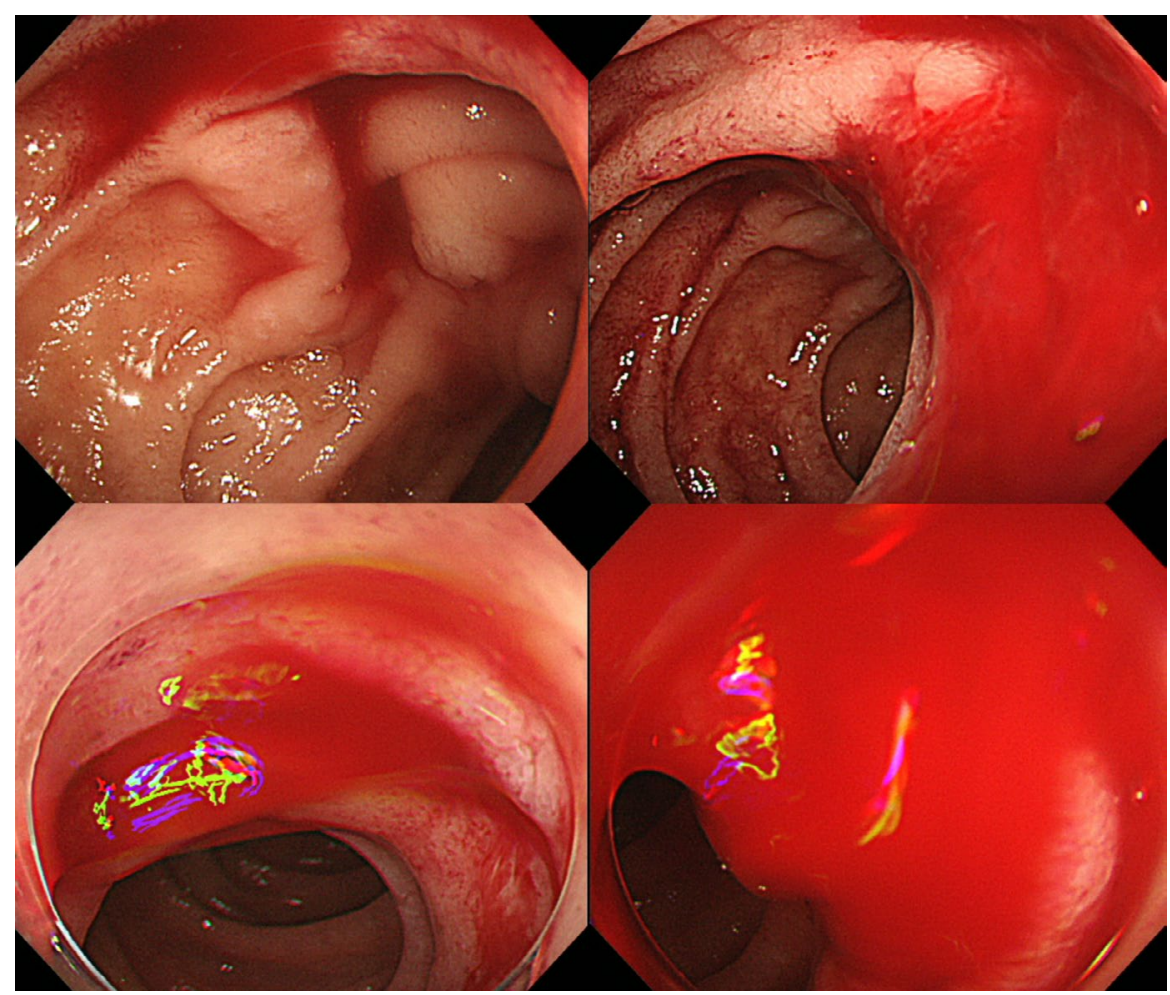

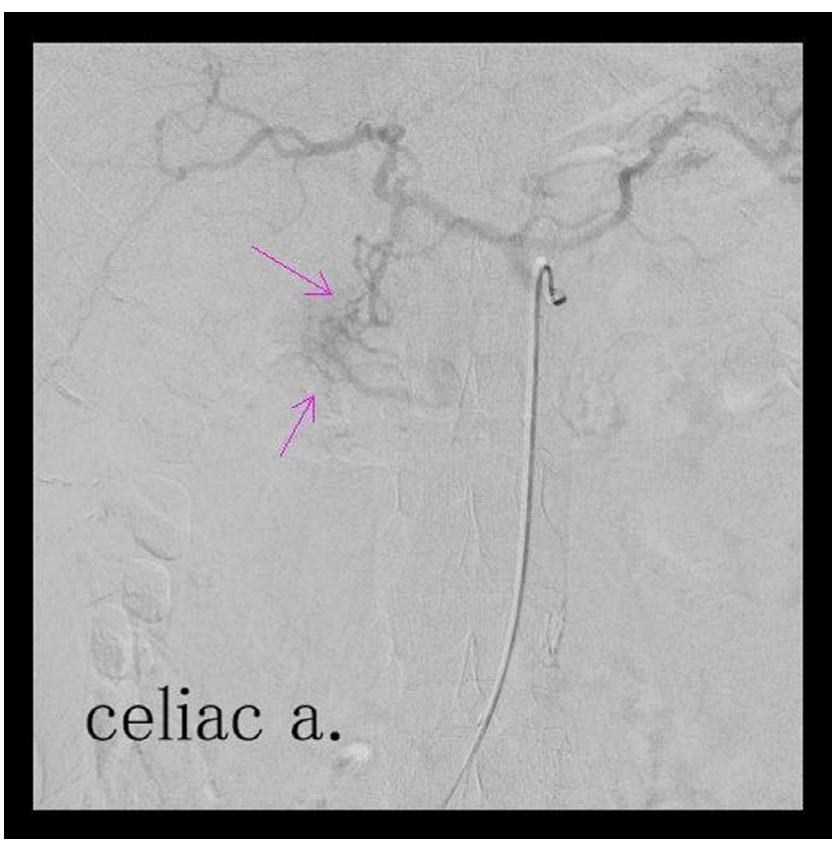

F I G U RE 3 Abdominal angiography: selective imaging of the gastroduodenal artery revealed abnormal reticular blood vessels in the descending limb of the duodenum

The most common symptoms were gastrointestinal bleeding (47\%) and epigastric pain (46\%). ${ }^{6}$ It was also seen that, in the pancreatic head AVM group, bleeding was the most common complication, while in the pancreatic body-tail AVM group, pancreatitis was more common. ${ }^{7}$

In our Ph-AVM case, the patient also had bleeding, but no pancreatitis. As a treatment, incomplete treatment of pancreatic AVM, such as arterial embolization, can result in growing new collateral vessels and cause the progression of portal hypertension, leading to recurrent gastrointestinal bleeding and rupture of the esophageal varices. ${ }^{7}$ Repeated gastrointestinal bleeding was observed in this case as well. The causes were esophagogastric varicose bleeding due to portal hypertension associated with AVM, rupture of AVM itself into the gastrointestinal tract, bleeding from an ulcer caused by an ischemic change of the gastrointestinal mucosa due to progression of AVM, bleeding of AVM into the pancreatic duct, and bleeding of AVM into the bile duct. ${ }^{8-10}$ In this case, no varicose veins were observed, no ulceration was formed, and bleeding from the papilla of Vater was not clear. Therefore, it is considered that the bleeding was caused by the mechanism of rupture of AVM itself into the gastrointestinal tract. Gastrointestinal ulcer (26.8\%) was the most common comorbidity of pancreatic AVM in 97 cases of pancreatic AVM reported by Hirai et al. in Japan. ${ }^{11}$ But it is unclear whether the history of this case treated for duodenal ulcer 20 years ago was a probable cause or not for patient's case. The AVM is located in the pancreatic head in more than half of the lesions (59.4\%), followed by the body and tail $(33.3 \%)$, and the entire pancreas $(7.2 \%)$ as this case. ${ }^{5}$

Contrast-enhanced CT and angiography are helpful for diagnosis. ${ }^{8}$ As a characterization of contrast-enhanced CT result, it is known that in the early stage of the arterial phase, there is reticular deep stain of lesions and depiction of the portal vein, ${ }^{3,12}$ as well as dilated and tortuous inflowing blood vessels, reticular intrapancreatic 


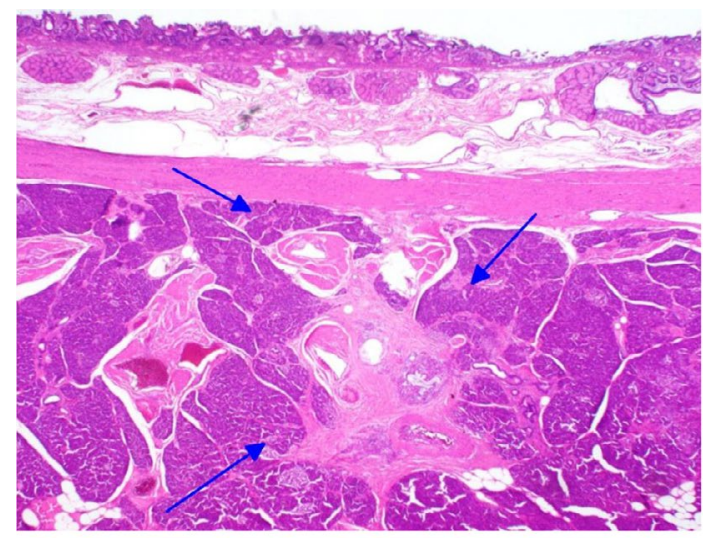

F I G URE 4 Histopathological findings: collections of irregularly tortuous blood vessels with the thick wall in the duodenal proper muscle layer adjacent to the pancreatic parenchyma near the major duodenal papilla

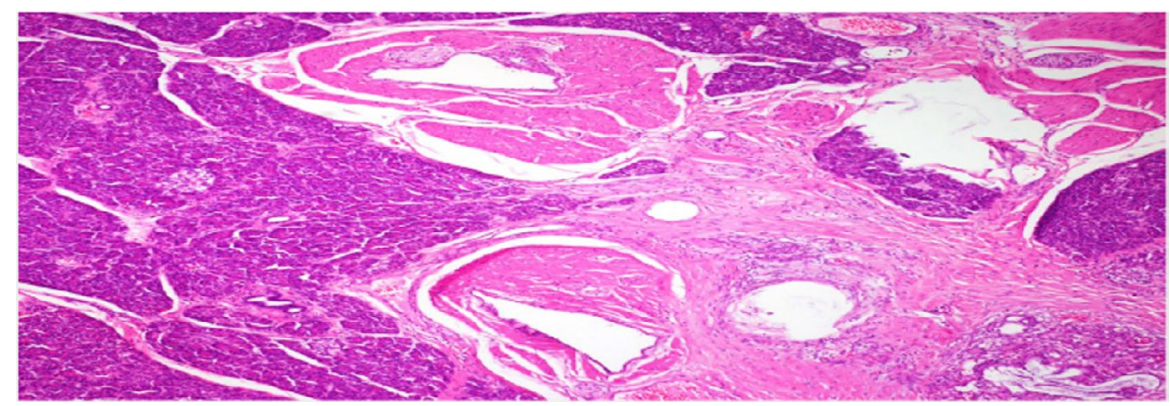

vascular plexus, and early venous return to portal vein or splenic vein are characteristic findings in angiography. In addition, there are reports that angiography is not only important for diagnosis, but also for obtaining detailed information such as multiple lesions, localization of lesions, and their spread as well. ${ }^{13}$ In this case, CT showed spotty staining of the pancreatic uncinate process, and angiography showed a reticulated vascular plexus at the pancreatic head, which was similar in characteristics.

According to the report by Hirai et $a 1,{ }^{11}$ pancreatic resection was performed in 46 patients (47.4\%) for treatment. Since there are many cases with lesions at the pancreatic head, pancreatoduodenectomy is performed most often, and it was performed in 30 cases (30.9\%). Minimally invasive treatment with interventional radiology may be selected due to poor general condition; nevertheless, surgery is selected as the radical treatment if there are many inflowing blood vessels and complicated collateral circulation because it is difficult to embolize all of them. In this case, as well, complete hemostasis was not achieved with coil embolization, therefore pancreatoduodenectomy was selected.

\section{CONCLUSION}

We experienced a case in which emergency pancreatoduodenectomy was performed for hemorrhagic shock due to pancreatic AVM, and the patient was saved. For this disease, surgical resection also should be another option besides noninvasive treatment if necessary.

\section{ACKNOWLEDGMENTS}

The authors would like to gratefully thank everyone who was involved in this work. The authors also thank Lupe Kimble, MSN, APRN-BC, NP-C, NPIII at Kaiser Permanente, Roseville, CA, and Andrew Kimble, Multiple Subject and English Single Subject, Educator, B.A. English UC Davis, CA, for their tremendous guidance and support with editing of this paper.

\section{CONFLICTS OF INTEREST}

The authors declare no conflicts of interest in association with this study.

\section{AUTHOR CONTRIBUTIONS}

MK drafted the manuscript. MF gathered the data and edited the draft. KK, NI, TO, ME, GK, RS, SU, RT, and TS participated in the critical revision of the manuscript. All authors read and approved the final manuscript.

\section{ETHICAL APPROVAL}

Informed consent was obtained from the patient regarding the report of his clinical scenario data in an anonymous way.

\section{CONSENT}

The patient has provided written consent for the case report to be published.

\section{DATA AVAILABILITY STATEMENT}

Data sharing is not applicable to this article as no datasets were generated or analyzed during the current study. 


\section{ORCID}

Masaki Kitazono (1) https://orcid.

org/0000-0001-9298-0189

\section{REFERENCES}

1. Halpern M, Turner AF, Citron BP. Hereditary hemorrhagic telangiectasia. An angiographic study of abdominal visceral angiodysplasias associated with gastrointestinal hemorrhage. Radiology. 1968;90:1143-1149.

2. Meyer CT, Troncale FJ, Galloway S, et al. Arteriovenous malformations of the bowel: an analysis of 22 cases and a review of the literature. Medicine. 1981;60:36-48.

3. Malan E, Puglionisi A. Congenital angiodysplasias of the extremities. I. Generalities and classification; venous dysplasias. $J$ Cardiovasc Surg. 1964;5:87-130.

4. Ishikawa T. Congenital arteriovenous malformations involving the pelvis and retroperitoneum: a case report. Angiology. 1979;30:70-74.

5. Song KB, Kim SC, Park JB, et al. Surgical outcomes of pancreatic arteriovenous malformation in a single center and review of literature. Pancreas. 2012;41:388-396.

6. Chou SC, Shyr YM, Wang SE. Pancreatic arteriovenous malformation. J Gastrointest Surg. 2013;17:1240-1246.

7. Park J, Park I, Kim M, Ryu H, Lee JK. Arteriovenous malformation with a retention cyst of the pancreas. Korean J Pancreas Biliary Tract. 2018;23(3):116-121.

8. Chuang VP, Pulmano CM, Walter JF, et al. Angiography of pancreatic arteriovenous malformation. Am J Roentogenol. 1977;129:1015-1018.
9. Yagyu T, Murayama M, Nakamura E, et al. A case of arteriovenous malformation of the pancreas head detected in conjunction with an intractable post-bulbar duodenal ulcer. J Jpn Surg Assoc. 2002;63:1523-1527.

10. Yamamoto H, Matsueda K, Wakiya I, et al. A case of pancreatic arteriovenous malformation causing duodenal hemorrhage. Gastroenterol Endosc. 1997;39:226-232.

11. Hirai T, Homma Y, Yabushita Y, et al. A case of pancreatic arteriovenous malformation with an associated pseudoaneurysm. $J$ Jpn Surg Assoc. 1998;31(9):12006-12010.

12. Matsumi $\mathrm{Y}$, Kobashi $\mathrm{K}$, Hatano $\mathrm{M}$, et al. A duodenal venous malformation treated by segmental duodenal resection after selective transcatheter arterial embolization. J Jpn Surg Assoc. 2017;78:508-514.

13. Moore JD, Thompson NW, Appelman HD, et al. Arteriovenous malformation of the gastrointestinal tract. Arch Surg. 1976;111:381-389.

How to cite this article: Kitazono M, Fujita M, Katsue K, et al. A case of emergency pancreatoduodenectomy for bleeding from the duodenal mucosa due to arteriovenous malformation of the pancreatic head. Clin Case Rep. 2021;9:e04824. https://doi.org/10.1002/ccr3.4824 